\title{
PREVALÊNCIA DA HIPERTENSÃO ARTERIAL E FATORES ASSOCIADOS EM IDOSOS
}

\author{
Prevalence of arterial hypertension and associated factors in \\ the elderly \\ Prevalencia de hipertensión arterial en mayores y los factores \\ asociados
}

Artigo Original

\section{RESUMO}

Objetivo: Investigar a prevalência de hipertensão arterial sistêmica (HAS) em idosos e verificar perfil sociodemográfico, fatores de risco e complicações dos idosos identificados com hipertensão. Métodos: Estudo descritivo, quantitativo, realizado com 220 prontuários de idosos em um Centro de Saúde de São Luís-MA, Brasil, entre 2011 e 2012. Por meio da aplicação de um formulário estruturado, coletaram-se dados dos prontuários e das fichas do Hiperdia, com variáveis sociodemográficas e clínicas. Realizou-se análise estatística descritiva com o Statistical Package for the Social Sciences e aplicou-se o teste qui-quadrado. Resultados: A prevalência da hipertensão entre os idosos foi de 51,4\% ( $\mathrm{n}=113$; IC95\%). Destes, 63,7\% $(n=72)$ eram do sexo feminino; 64,6\% $(n=73)$, sedentários; $52,2 \%(n=59)$ tinham sobrepeso; 53,1\% ( $n=60)$, obesidade abdominal; 29,2\% ( $n=33)$ eram diabéticos; $17,7 \%(n=20)$ apresentavam comorbidades e complicações; 79,6\% $(n=90)$ estavam com a pressão arterial aumentada na primeira consulta; e $66,6 \%(n=60)$, na última. Conclusão: A HAS apresentou alta prevalência nos idosos investigados, sendo maior em determinados subgrupos: mulheres, baixa escolaridade e não brancos. Dentre os fatores de risco mais comuns, destacaram-se o sedentarismo, o sobrepeso e a obesidade abdominal.

Descritores: Idoso; Hipertensão; Prevalência.

\section{ABSTRACT}

Objective: To investigate the prevalence of systemic hypertension among the elderly and verify the sociodemographic profile, risk factors and complications of elderly individuals identified with hypertension. Methods: Quantitative descriptive study, performed with 220 medical records of elderly patients in a Health Center in São Luis, Maranhão, Brazil, between 2011 and 2012. By applying a structured questionnaire containing sociodemographic and clinical variables, data was collected from the medical records and Hiperdia files. Descriptive statistical analysis was performed with the Statistical Package for the Social Sciences and the chi-square test was applied. Results: The prevalence of hypertension in the elderly was $51.4 \%(n=113$; IC95\%). Of these, $63.7 \%(n=72)$ were female; $64.6 \%(n=73)$ were sedentary; $52.2 \%(n=59)$ were overweight; $53.1 \%(n=60)$ had abdominal obesity; $29.2 \%(n=33)$ had diabetes; $17.7 \%(n=20)$ had comorbidities and complications; $79.6 \%(n=90)$ were found with increased blood pressure in the first consultation, and $66.6 \%(n=60)$, in the last one. Conclusion: The prevalence of hypertension was high among the elderly assessed, being higher in some subgroups: women, low level of education, and non-white. Among the most common risk factors, the sedentary lifestyle, overweight, and abdominal obesity stand out.

Descriptors: Aged; Hypertension; Prevalence.

Recebido em: 18/11/2013 Revisado em: 01/02/2014 Aceito em: 05/07/2014

1) Universidade Federal do Maranhão UFMA - São Luís (MA) - Brasil

2) Universidade Federal do Ceará -UFC Fortaleza (CE) - Brasil

3) Universidade Estadual do Ceará - UECE - Fortaleza (CE) - Brasil

4) Universidade da Integração Internacional da Lusofonia Afro-Brasileira - UNILAB Redenção (CE) - Brasil 


\section{RESUMEN}

Objetivo: Investigar la prevalencia de hipertensión arterial sistémica (HAS) en mayores y verificar el perfil sociodemográfico, factores de riesgo y complicaciones de los mayores identificados con hipertensión. Métodos: Estudio descriptivo y cuantitativo realizado en 220 historiales clínicos de mayores de un Centro de Salud de São Luiz-MA, Brasil, entre 2011 y 2012. Se recogieron datos de los historiales clínicos y fichas del Hiperdía a través de un formulario estructurado con variables sociodemograficas y clínicas. Se realizó el análisis estadístico descriptivo con el Statistical Package for the Social Sciences y la aplicación de la prueba de Chi-cuadrado. Resultados: La prevalencia de hipertensión en los mayores fue del 51,4\% ( $n=113$; IC95\%). De ellos, el 63,7\% (n=72) eran del sexo femenino; el 64,6\% $(n=73)$ sedentarios; el 52,2\% $(n=59)$ tenían sobrepeso; el 53,1\% $(n=60)$ obesidad abdominal; el 29,2\% (n=33) eran diabéticos; el $17,7 \%(n=20)$ presentaban comorbidades y complicaciones; el $79,6 \%(n=90)$ presentó la presión arterial elevada en la primera consulta; y el 66,6\% $(n=60)$ en la última. Conclusión: La HAS presento elevada prevalencia en los mayores investigados, siendo la presión arterial mayor en determinados grupos: mujeres, escolaridad baja, y mayores no blancos. De los factores de riesgo más comunes se destacaron el sedentarismo, el sobrepeso y la obesidad abdominal.

Descriptores: Anciano; Hipertensión; Prevalencia.

\section{INTRODUÇÃO}

No cenário brasileiro e mundial, a hipertensão arterial sistêmica (HAS) tem se destacado devido à alta incidência e prevalência. Essa doença ocorre quando o sangue circula com alta pressão nos vasos - igual ou acima de 140x90mmHg. Seu desenvolvimento é multifatorial; dentre os fatores, estão: idade, sexo, etnia, sobrepeso/obesidade, ingesta de sal, ingesta de álcool, sedentarismo, fatores socioeconômicos e genética ${ }^{(1)}$.

Ela pode acometer qualquer faixa etária, mas se verifica na população idosa um elevado número de portadores de HAS devido às mudanças orgânicas que o envelhecimento proporciona. Sabe-se que, ao longo do envelhecimento, surgem alterações morfológicas (artérias enrijecidas), metabólicas e psíquicas que contribuem para o aumento da pressão $\operatorname{arterial}^{(2)}$.

A HAS é um problema de saúde pública no Brasil e no mundo, pois nos últimos 20 anos mostrou prevalência superior a 30\%, e no período de 2003 a 2008, 44 estudos em 35 países revelaram uma prevalência global de $37,8 \%$ em homens e $32,1 \%$ em mulheres ${ }^{(1)}$. Além disso, a HAS é fator de risco para doenças cardiovasculares, cerebrovasculares e insuficiência renal crônica, sendo causadora de $40 \%$ das mortes por acidente vascular cerebral (AVC), 25\% das mortes por doença arterial coronariana e, juntamente com o diabetes, representa $62,1 \%$ do diagnóstico primário de pessoas submetidas à diálise ${ }^{(3)}$.

Estudos revelam que, dentre os idosos brasileiros (> 65 anos), mais de $60 \%$ são hipertensos ${ }^{(4)}$. Porém, as informações de estudos epidemiológicos sobre a população idosa são raras ou pouco divulgadas no Brasil até o ano de $2008^{(2)}$. Além disso, a população idosa representa $12 \%$ da população brasileira, dado verificado no último Censo, em $2010^{(5)}$. Dessa forma, é necessário conhecer a real situação de saúde dos idosos e sua demanda pelos serviços médicos, para oferecer subsídios no planejamento de ações preventivas e curativas, reduzindo a morbimortalidade e proporcionando melhor qualidade de vida para os idosos. Nesse sentido, a atenção básica ganha destaque por priorizar ações de promoção da saúde e prevenção de doenças, tornando-se peça fundamental para controlar a incidência e prevalência da hipertensão em idosos ${ }^{(2)}$.

Diante do contexto apresentado, o objetivo do estudo foi investigar a prevalência da HAS em idosos e verificar perfil sociodemográfico, fatores de risco e complicações dos idosos identificados com hipertensão.

\section{MÉTODOS}

Pesquisa de natureza descritiva, com abordagem quantitativa, realizada com idosos -

indivíduos com 60 anos ou mais ${ }^{(6)}$ - devidamente cadastrados em um Centro de Saúde no município de São Luís-MA, Brasil. A cidade abrange quatro equipes da Estratégia Saúde da Família (ESF), totalizando mais de quatro mil famílias cobertas, além do atendimento de áreas descobertas. Selecionou-se intencionalmente uma equipe para o estudo, que possuía 270 idosos cadastrados. A casuística foi calculada baseada na fórmula estatística para populações finitas, com nível de confiança de 95\%, resultando em uma amostra de $220 \operatorname{idosos}^{(7)}$.

Incluíram-se prontuários de indivíduos com idade igual ou superior a 60 anos, independentemente do sexo, e hipertensos cadastrados no programa Hiperdia, do Centro de Saúde, no período de 2005 a 2011. Seriam excluídos os pacientes cujos prontuários estivessem com preenchimento insuficiente dos dados, mas não houve ninguém nessa situação. A seleção dos prontuários ocorreu de forma aleatória, através do sorteio da numeração.

Os dados foram coletados nos prontuários e nas fichas do Hiperdia, na sala de recepção da Unidade Básica de Saúde, de outubro de 2011 a janeiro de 2012.

Por meio de um formulário estruturado, coletaramse variáveis sociodemográficas (idade, sexo, raça, escolaridade), antropométricas [peso, altura, Índice de Massa Corpórea (IMC), circunferência abdominal (CA)], 
fatores de risco [sedentarismo, tabagismo, antecedentes familiares cardiovasculares (AFCV)], pressão arterial (PA), medicações, diabetes melitus e complicações, incluindo acidente vascular cerebral (AVC), doença renal e miocardiopatia.

O IMC foi calculado de acordo com a equação de Ketelet $^{(8)}(\mathrm{IMC}=\mathrm{kg} / \mathrm{m} 2)$, que utiliza pontos de corte próprios para o idoso, segundo a recomendação do Ministério da Saúde: baixo peso, $\leq 22$, eutrófico $>22$ e $<27$ e sobrepeso $\geq$ 27. Considerou-se a CA aumentada - obesidade abdominal - através de pontos de corte recomendados pela Organização Mundial de Saúde $(\mathrm{OMS})^{(9)}$ : CA $\geq 102 \mathrm{~cm}$ para homens e $\mathrm{CA} \geq 88 \mathrm{~cm}$ para mulheres. A PA, segundo as VI Diretrizes Brasileiras de Hipertensão, recebeu a classificação de: ótima: $<120$ x 80mmHg; boa: < 130 x $85 \mathrm{mmHg}$; limitrofe: 130-139 x 85-89mmHg; hipertensão estágio I: 140-159 x 90-99mmHg; hipertensão estágio II: 160-179 x 100109mmHg; hipertensão estágio III: $\geq 180 \times 110 \mathrm{mmHg}$; e hipertensão sistólica isolada: $\geq 140 \mathrm{x}<90 \mathrm{mmHg}^{(1)}$.

Através de análise estatística descritiva e do Statistical Package for the Social Sciences (SPSS), versão 18.0 , verificou-se a associação entre as variáveis sociodemográficas e os fatores de risco com valor da PA e do IMC por meio do teste do qui-quadrado.

A pesquisa cumpriu os princípios éticos da Resolução $466 / 12^{(10)}$ do Conselho Nacional de Saúde no que se refere à pesquisa que envolve seres humanos. Este estudo faz parte de um projeto integrado, aprovado pelo Comitê de Ética em Pesquisa da Universidade Federal do Maranhão (UFMA), sob parecer $n^{\circ} 150 / 2011$.

\section{RESULTADOS}

Dos 220 prontuários analisados, identificaram-se 113 hipertensos, representando a prevalência de 51,4\% (IC95\%: $44,8 \%-58 \%$ ) dos idosos.

Dos dados dos 113 hipertensos utilizados para a análise estatística, as mulheres obtiveram maior prevalência da HAS que os homens, representando $63,7 \%(\mathrm{n}=72)$ dos idosos hipertensos. A faixa etária com maior número de idosos foi de 70 a 79 anos, com $31 \%$ (n=35), e média de idade de 72,7 anos ( $\mathrm{DP}=72,7 \pm 8,9)$. A maioria, $32,7 \%$ $(\mathrm{n}=37)$, tinha o nível fundamental incompleto e $65,5 \%$ $(n=74)$ eram não brancos (Tabela I).

Tabela I - Distribuição dos idosos hipertensos segundo o perfil sociodemográfico. São Luís-MA, 2011.

\begin{tabular}{lccc}
\hline Características & $\boldsymbol{n}$ & $\mathbf{\%}$ & \\
\hline Sexo & 72 & 63,7 & Média \pm DP \\
$\quad$ Feminino & 41 & 36,3 & $72,7 \pm 8,9$ \\
$\quad$ Masculino & & & 17,7 \\
Idade (anos) & 20 & 26,5 & 31 \\
$60-64$ & 30 & 24,8 & \\
$65-69$ & 35 & 26,6 \\
$70-79$ & 28 & 29,2 \\
$\geq 80$ & & 32,7 \\
Escolaridade & 30 & 3,5 \\
Não sabe ler & 33 & 1,8 \\
Alfabetizado & 37 & 1,8 \\
Fundamental incompleto & 4 & 4,4 \\
Fundamental completo & 2 & \\
Médio incompleto & 2 & 33,6 \\
Médio completo & 5 & 14,2 \\
Não informa & & 2,7 \\
Raça & 38 & 45,1 \\
Branca & 16 & 3,5 \\
Preta & 3 & 0,9 \\
Amarela & 51 & 4 \\
Parda & 1 & \\
Indígena & & \\
Não informa & & \\
\hline
\end{tabular}


Dentre os idosos hipertensos, $15,9 \%(n=18)$ tinham $\mathrm{AFCV}$, a maioria era sedentária, $64,6 \%(\mathrm{n}=73)$, e uma parcela era tabagista, $12,4 \%(\mathrm{n}=14)$. Grande parte tinha sobrepeso, $52,2 \%(\mathrm{n}=59)$, com média de IMC de 27,7. A obesidade abdominal foi encontrada em 53,1\% ( $\mathrm{n}=60)$, com a média de $92,2 \mathrm{~cm}$ de circunferência abdominal.

$\mathrm{O}$ total de idosos hipertensos com diabetes representou $29,2 \%(n=33)$. A presença de comorbidades e complicações foram relatadas em 17,7\% $(\mathrm{n}=20)$, sendo o AVC e a doença renal os mais prevalentes, com $40 \%(\mathrm{n}=8)$ e $25 \%(\mathrm{n}=5)$, respectivamente (Tabela II).

As medicações mais usadas foram a Hidroclorotiazida (HCTZ) $(54,8 \%, n=62)$, o Captopril $(50,4 \%, n=57)$ e o Propanolol $(19,5 \%, \mathrm{n}=22)$. Os pacientes que fizeram uso de monoterapia somaram $23 \%(\mathrm{n}=26)$, utilizando com maior prevalência o Captopril e a HCTZ. A terapia mais utilizada foi a combinada, em $64,6 \%(n=73)$ dos pacientes. Um total de $12,4 \%(n=14)$ dos pacientes abandonaram o tratamento ou não deram seguimento no Centro de Saúde.

Identificou-se melhora dos níveis pressóricos entre a primeira e a última consulta, considerando que 79,6\% $(\mathrm{n}=90)$ estavam com a PA alterada $(\geq 140 / 90 \mathrm{mmHg})$ na primeira consulta e na última esse valor baixou para $66,6 \%$ $(\mathrm{n}=60)$. Observou-se que os pacientes com 80 anos ou mais tiveram a melhor evolução, com queda de quase $30 \%$ da PA, apesar de os níveis pressóricos ainda estarem elevados na última consulta. Com relação ao sexo, ambos tiveram queda nos níveis pressóricos, mas o sexo masculino apresentou maior dificuldade no controle da PA. Quanto à raça/cor e escolaridade, houve uma evolução positiva e similar dos níveis da pressão arterial. Os pacientes não diabéticos estavam com a PA mais aumentada que os diabéticos na primeira consulta, mas a evolução da PA dos não diabéticos foi mais favorável. Pacientes com comorbidade tiveram

Tabela II - Distribuição dos idosos hipertensos segundo o perfil clínico. São Luís-MA, 2011.

\begin{tabular}{|c|c|c|c|c|}
\hline Características & $n$ & $\%$ & & \\
\hline \multicolumn{5}{|l|}{ Fatores de risco } \\
\hline $\mathrm{AFCV}$ & 18 & 15,9 & & \\
\hline Sedentarismo & 73 & 64,6 & & \\
\hline Tabagismo & 14 & 12,4 & & \\
\hline $\mathrm{IMC}$ & & & \multicolumn{2}{|c|}{ Média \pm DP } \\
\hline Baixo peso & 10 & 8,8 & \multicolumn{2}{|c|}{$27,7 \pm 4,4$} \\
\hline Eutrófico & 43 & 38,1 & & \\
\hline Sobrepeso & 59 & 52,2 & \multirow{2}{*}{\multicolumn{2}{|c|}{ Média \pm DP }} \\
\hline Cintura abdominal & & & & \\
\hline Alterada & 60 & 53,1 & \multicolumn{2}{|c|}{$92,2 \pm 16,9$} \\
\hline Diabetes & 33 & 29,2 & & \\
\hline \multicolumn{5}{|c|}{ Comorbidades/Complicações } \\
\hline Sim & 20 & 17,7 & & \\
\hline AVC & 8 & 40 & & \\
\hline Doença renal & 5 & 25 & & \\
\hline Miocardiopatia & 2 & 10 & & \\
\hline Outras & 5 & 25 & & \\
\hline Medicações & $\mathbf{I}^{*}$ & $A^{* *}$ & Total & $\%$ \\
\hline Hidroclorotiazida & 6 & 56 & 62 & 54,8 \\
\hline Captopril & 14 & 43 & 57 & 50,4 \\
\hline Propanolol & 1 & 21 & 22 & 19,5 \\
\hline Outros & 5 & 32 & 37 & 32,8 \\
\hline TOTAL & 26 & 152 & 178 & \\
\hline Sem medicação ${ }^{* * *}$ & - & - & 11 & \\
\hline Sem seguimento ${ }^{* * * *}$ & - & - & 3 & \\
\hline
\end{tabular}

AFCV: Antecedentes Familiares Cardiovasculares; IMC: Índice de Massa Corpórea; AVC: Acidente Vascular Cerebral; "Uso isolado; *Uso associado; ${ }^{* * *}$ Não usavam nenhuma medicação na última consulta; ${ }^{* * * *}$ Não acompanharam as consultas do Hiperdia. 
uma redução da PA na última consulta de 29,5\% (n=6), e aqueles sem comorbidade tiveram 17,3\% $(\mathrm{n}=24)$ (Tabela III).

$\mathrm{Na}$ primeira consulta, $1,8 \% \quad(\mathrm{n}=2)$ tinham a PA considerada ótima; $8 \%(\mathrm{n}=9)$ eram classificados como boa; $10,6 \%(n=12)$, como limítrofe; $13,3 \%(n=15)$, como hipertensão estágio I; $15 \%(\mathrm{n}=17)$, como hipertensão estágio II; 16,8\% ( $\mathrm{n}=19)$, como hipertensão estágio III; e $34,6 \%$ ( $\mathrm{n}=39)$, como hipertensão sistólica isolada.
O estudo revelou alta prevalência de sobrepeso nos idosos hipertensos, com maior proporção para o sexo feminino $(56,9 \%, \mathrm{n}=41)$ em comparação ao masculino $(43,9 \%, n=18)$. Dentre os pacientes com sobrepeso, $56,9 \%$ $(n=41)$ eram sedentários, $75 \%(n=45)$ tinham obesidade abdominal, $66,7 \%(n=22)$ eram diabéticos e $53,8 \%(n=50)$ não apresentavam comorbidades - estes numa proporção maior do que aqueles com alguma comorbidade (Tabela IV).

Tabela III - Distribuição dos idosos hipertensos com pressão arterial superior a 140 X 90 mmHg durante primeira e última consulta. São Luís-MA, 2011.

\begin{tabular}{|c|c|c|c|c|c|c|}
\hline \multirow{3}{*}{ Características } & \multicolumn{6}{|c|}{ PRESSÃO ARTERIAL $\geq 140$ X 90 mmHg } \\
\hline & \multicolumn{3}{|c|}{ Primeira Consulta* } & \multicolumn{3}{|c|}{ Última Consulta** } \\
\hline & $n$ & $\%$ & $p$ & $n$ & $\%$ & $p$ \\
\hline Idade & & & 0,020 & & & 0,186 \\
\hline $60-64$ & 12 & 60,0 & & 6 & 37,5 & \\
\hline $65-69$ & 23 & 76,7 & & 19 & 67,9 & \\
\hline $70-79$ & 28 & 80,0 & & 18 & 58,1 & \\
\hline$\geq 80$ & 27 & 96,4 & & 17 & 68,0 & \\
\hline Sexo & & & 0,035 & & & 0,024 \\
\hline Masculino & 37 & 90,2 & & 25 & 75,8 & \\
\hline Feminino & 53 & 73,6 & & 35 & 52,2 & \\
\hline Raça/Cor & & & 0,597 & & & 0,931 \\
\hline Branca & 28 & 73,7 & & 18 & 58,1 & \\
\hline Preta & 12 & 75,0 & & 8 & 53,3 & \\
\hline Amarela/Índigena & 6 & 85,7 & & 3 & 60,0 & \\
\hline Parda & 43 & 84,3 & & 30 & 62,5 & \\
\hline Escolaridade & & & 0,439 & & & 0,244 \\
\hline Não sabe ler & 25 & 83,3 & & 18 & 66,7 & \\
\hline Alfabetizado & 26 & 78,8 & & 18 & 54,5 & \\
\hline Fund. Incompleto & 28 & 75,7 & & 18 & 56,3 & \\
\hline$\geq$ Fund. Completo & 8 & 100 & & 3 & 100 & \\
\hline Não informa & 3 & 60 & & 3 & 60 & \\
\hline Diabetes & & & 0,092 & & & 0,480 \\
\hline Sim & 23 & 69,7 & & 17 & 54,8 & \\
\hline Não & 67 & 83,8 & & 43 & 62,3 & \\
\hline Comorbidades & & & 0,183 & & & 0,043 \\
\hline Sim & 13 & 68,4 & & 7 & 38,9 & \\
\hline Não & 77 & 81,9 & & 53 & 64,6 & \\
\hline
\end{tabular}

AFCV: Antecedentes Familiares Cardiovasculares; ${ }^{*} \mathrm{n}=90 ;{ }^{* *} \mathrm{n}=60$. 
Tabela IV - Distribuição dos idosos hipertensos segundo o Índice de massa corporal IMC e características. São Luís-MA, 2011.

\begin{tabular}{|c|c|c|c|c|c|c|c|}
\hline \multirow{3}{*}{ Características } & \multicolumn{6}{|c|}{ Índice de massa corpórea - IMC } & \multirow[b]{3}{*}{$p$} \\
\hline & \multicolumn{2}{|c|}{ Baixo peso } & \multicolumn{2}{|c|}{ Eutrófico } & \multicolumn{2}{|c|}{ Sobrepeso } & \\
\hline & n & $\%$ & $\mathbf{n}$ & $\%$ & $\bar{n}$ & $\%$ & \\
\hline \multicolumn{8}{|l|}{ Sexo } \\
\hline \multirow{2}{*}{$\begin{array}{l}\text { Masculino } \\
\text { Feminino }\end{array}$} & 5 & 12,2 & 18 & 43,9 & 18 & 43,9 & \multirow[t]{2}{*}{0,328} \\
\hline & 5 & 6,9 & 26 & 36,1 & 41 & 56,9 & \\
\hline Sedentarismo & 7 & 9,7 & 24 & 33,3 & 41 & 56,9 & 0,336 \\
\hline Obesidade abdominal & 0 & 0,0 & 15 & 25,0 & 45 & 75,0 & $<0,0001$ \\
\hline Diabetes & 1 & 3,0 & 10 & 30,3 & 22 & 66,7 & 0,113 \\
\hline \multicolumn{8}{|l|}{ Comorbidades } \\
\hline Sim & 1 & 5,3 & 9 & 47,4 & 9 & 47,4 & 0,623 \\
\hline Não & 9 & 9,7 & 34 & 36,5 & 50 & 53,8 & \\
\hline
\end{tabular}

\section{DISCUSSÃO}

A prevalência da HAS em idosos (51,4\%) foi semelhante à de outra pesquisa de análise de prontuários desenvolvida numa UBS em Marcelino Ramos-RS ${ }^{(11)}$. Contudo, em comparação a um estudo em Fortaleza-CE, a prevalência foi expressivamente inferior, $68,6 \%{ }^{(12)}$. Com base nos dados do DATASUS, que considerou a faixa etária de 65 anos e mais, a prevalência também foi inferior em relação à população idosa brasileira, $63,2 \%$, e a ludovicense, $57,2 \%{ }^{(13)}$

$\mathrm{Na}$ presente pesquisa, houve maior prevalência da HAS no sexo feminino, resultado similar ao de outros estudos $^{(14,15)}$. Isso ocorre porque a prevalência global de HAS entre homens e mulheres é mais elevada nestas após os 50 anos $^{(1)}$. Além disso, a mulher tem uma longevidade maior e procura mais os serviços de saúde que os homens, favorecendo o diagnóstico de $\mathrm{HAS}^{(12)}$.

Com relação à escolaridade, encontrou-se maior prevalência da HAS em idosos com menor escolaridade. Esses dados são compatíveis com a prevalência nacional e com pesquisas que detectaram um perfil educacional ruim nos idosos brasileiros ${ }^{(1,16)}$. Entretanto, é complexo estabelecer esses dados ${ }^{(1)}$, considerando que a presente pesquisa foi desenvolvida numa comunidade periférica, onde o nível de instrução é baixo.

A maioria dos hipertensos era de não brancos, confirmando pesquisa que verificou que a HAS é duas vezes mais prevalente em indivíduos de cor não branca ${ }^{(1)}$. Porém, não se conhece com exatidão o impacto da miscigenação sobre a HAS no Brasil ${ }^{(1)}$.

O AFCV, fator de risco não modificável, teve uma prevalência relativamente baixa $(15,9 \%)$ em comparação com outra pesquisa realizada com hipertensos cadastrados no Hiperdia num município do Paraná ${ }^{(17)}$, onde se verificou que a maioria possuía antecedentes familiares para HAS (70\%). Em outro estudo com idosos da periferia de Fortaleza-CE, 59,3\% apresentaram $\mathrm{AFCV}^{(2)}$. A baixa prevalência desse fator de risco no presente estudo pode ter sido por subnotificação dos dados, pois há a possibilidade de a população pesquisada não ter informações precisas quanto ao estado de saúde de seus antecedentes.

$\mathrm{O}$ sedentarismo representou o fator de risco mais prevalente na presente pesquisa, em $64,6 \%$ dos idosos hipertensos, corroborando com estudo transversal que teve amostra de 2.100 idosos ( $\geq 65$ anos) na região Nordeste, no qual a prevalência foi de $67,5 \%{ }^{(18)}$. A atividade física tem importância na manutenção da saúde, porém, tem se reduzido muito nas sociedades modernas, principalmente nos grupos de menor nível socioeconômico e com menor nível de escolaridade ${ }^{(19)}$.

Encontrou-se na atual investigação a prevalência do tabagismo de $12,4 \%$, similar a um estudo com idosos de 65 anos ou mais das regiões Sul e Nordeste do país, sem diagnóstico de hipertensão, o qual obteve uma prevalência de $15,2 \%{ }^{(18)}$.

Com relação ao IMC, a maioria dos idosos hipertensos da atual pesquisa apresentava sobrepeso, correspondendo a $52,2 \%$. Dados similares foram encontrados numa pesquisa com idosos hipertensos e diabéticos cadastrados no Hiperdia em Pelotas-RS, no qual se observou sobrepeso em 52,3\% da amostra $^{(20)}$. Quanto ao gênero, na atual pesquisa, as mulheres estavam significativamente com mais sobrepeso que os homens. Em duas pesquisas realizadas, também se 
observou o sobrepeso nas mulheres em níveis superiores aos homens ${ }^{(20,21)}$. Pesquisadores da Duke University Medical Center descobriram que as mulheres têm $48 \%$ mais chances de desenvolver obesidade que os homens. A razão para essa diferença ainda não foi bem entendida, mas se descobriu que a tendência natural de ganhar mais peso do que os homens pode explicar parte dessa diferença ${ }^{(22)}$.

A obesidade abdominal teve alta prevalência na presente pesquisa, em 53,6\% dos pacientes. Em outro estudo envolvendo idosos hipertensos cadastrados em uma Unidade de Saúde da Família de Londrina-PR, a prevalência foi ainda maior, $64,3 \%^{(21)}$, o que pode ser justificado devido ao aumento esperado do tecido gorduroso abdominal no processo de envelhecimento ${ }^{(21)}$.

O sedentarismo e a obesidade apresentaram bastante associação entre si na presente pesquisa. Dado verificado em estudo realizado no Paraná, com 53 portadores de HA estágio I, observou reduções significativas da variável IMC após seis meses de exercícios ${ }^{(23)}$.

No presente estudo, $29,2 \%$ dos idosos hipertensos eram diabéticos, dado superior ao de uma pesquisa realizada no município de Marcelino Ramos-RS, onde se encontrou a prevalência de $7,5 \%^{(11)}$.

$\mathrm{O}$ uso de medicações anti-hipertensivas tem como principal objetivo a redução da morbimortalidade cardiovascular, visando, assim, não apenas diminuir a pressão $\operatorname{arterial}^{(1)}$. Na população do presente estudo, a HCTZ, o Captopril e o Propanolol representaram as medicações mais prescritas, entretanto, em monoterapia, o Captopril e a HCTZ foram as drogas mais utilizadas, sendo a terapia combinada $(64,6 \%)$ mais prevalente que a monoterapia (23\%). A maioria dos idosos necessita de terapia combinada para o controle adequado da PA sistólica, porque evidências revelam que em $2 / 3$ dos pacientes a monoterapia não é suficiente para atingir as reduções de pressão previstas, além dos estágios elevados em que os idosos se encontraram na primeira consulta, indicando o uso inicial da terapia combinada para o controle da pressão ${ }^{(1)}$.

Um dos motivos pelos quais as drogas citadas têm alta prevalência no tratamento anti-hipertensivo no Centro de Saúde da pesquisa em questão é que esses medicamentos são disponibilizados gratuitamente pelo Sistema Único de Saúde, conforme a Portaria $\mathrm{n}^{\circ} 2.982$, de 26 de novembro de $2009^{(24)}$. Os governos federal, estadual e municipal referenciaram esses e outros medicamentos para a atenção básica no tratamento da HAS.

O abandono do tratamento farmacológico e a descontinuidade do seguimento dos idosos hipertensos foram destacados na presente e também em outra pesquisa, a qual encontrou uma prevalência de não adesão ao Programa Hiperdia de 8,5\%. Dentre os fatores relacionados, estavam o desconhecimento dos idosos e familiares sobre a doença e a pequena participação da família na condução do tratamento da hipertensão ${ }^{(25)}$.

Observou-se uma redução importante da PA entre a primeira e a última consulta. Pesquisa feita com hipertensos numa Unidade Básica de Saúde em Salvador-BA observou que $\operatorname{logo}$ após a introdução do tratamento, $28,9 \%$ dos hipertensos tinham a PA controlada e $57 \%$, no final do período observacional ${ }^{(26)}$. As mulheres tiveram um melhor controle da PA em relação aos homens, possivelmente porque possuem menor exposição a fatores de riscos, como tabagismo e etilismo, além das diferenças de atitude entre homens e mulheres quanto ao controle e tratamento das doenças $^{(12)}$. Pacientes com alguma comorbidade tinham melhor controle da PA no presente estudo. Isso é observado em pesquisas com idosos $\geq 80$ anos na Espanha, e com idosos $\geq 65$ anos na França, considerando que podem ser tratados mais agressivamente sob essas circunstâncias, já que, de uma maneira geral, os médicos são relutantes em tratar os pacientes idosos mais agressivamente, devido aos menores benefícios percebidos ou ao possível risco aumentado de eventos adversos ${ }^{(27)}$.

Percebeu-se que os idosos hipertensos se encontram no estágio da HAS mais avançado, sendo proporcional ao aumento da faixa etária. Além disso, uma grande porcentagem $(34,5 \%)$ tinha hipertensão sistólica isolada aumento da PA sistólica sem aumento da PA diastólica. Isso se dá porque a pressão sistólica aumenta continuamente com a idade; em contrapartida, a pressão diastólica aumenta até os 60 anos, diminuindo a seguir ${ }^{(28)}$. Segundo dados de 30 anos de seguimento de um estudo, isso é extremamente prejudicial, devido ao aumento do risco de eventos cardiovasculares com a elevação da pressão sistólica, particularmente no idoso ${ }^{(28)}$.

$\mathrm{Na}$ presente pesquisa, o sobrepeso esteve presente em maior quantidade nos pacientes sem comorbidade, em comparação aos que tinham alguma comorbidade. Estudo mostra que isso pode ser justificado pelo comprometimento do cuidador - mais presente em pacientes com comorbidade - em influenciar na adesão ao tratamento ${ }^{(29)}$.

A busca do perfil sociodemográfico e clínico dos pacientes apresentou limitações, pois o formulário de cadastro do Hiperdia não informa alguns dados relevantes para a pesquisa, como a situação socioeconômica, a ocupação e a ocorrência de outras doenças crônicas.

Sugere-se que se deve dar atenção especial para os hipertensos idosos, principalmente para os subgrupos mais vulneráveis, buscando contribuir para o controle da hipertensão arterial e a saúde do idoso, intensificando ações de promoção da saúde, prevenção de agravos, diagnóstico, tratamento e manutenção da saúde. 


\section{CONCLUSÃO}

A hipertensão arterial (HAS) apresentou alta prevalência nos idosos investigados, sendo maior em determinados subgrupos: mulheres, baixa escolaridade e não brancos. Dentre os fatores de risco mais comuns, destacaram-se o sedentarismo, o sobrepeso e a obesidade abdominal. As complicações mais frequentes identificadas nos idosos foram o acidente vascular cerebral e a doença renal. Por outro lado, percebeu-se uma melhora na evolução da pressão arterial após o início do tratamento clínico.

\section{AGRADECIMENTOS}

Ao Programa de Educação pelo Trabalho para a Saúde (PET-Saúde/Saúde da Família/UFMA).

\section{REFERÊNCIAS}

1. Sociedade Brasileira de Hipertensão. Diretrizes Brasileira de Hipertensão VI. Rev Bras Hipertens. 2010;17(1):1-64.

2. Caetano JA, Costa AC, Santos ZMSA, Soares E. Descrição dos fatores de risco para alterações cardiovasculares em um grupo de idosos. Texto \& Contexto Enferm. 2008; 17(2):327-35.

3. Ministério da Saúde (BR), Secretaria de Atenção à Saúde, Departamento de Atenção Básica. Envelhecimento e saúde da pessoa idosa. Brasília: Ministério da Saúde; 2006.

4. Cesarino CB, Cipullo JP, Martin JFV, Ciorlia LA, Godoy MRP, Cordeiro JA, et al. Prevalência e fatores sociodemográficos em hipertensos de São José do Rio Preto. Arq Bras Cardiol. 2008;91(1):31-5.

5. Instituto Brasileiro de Geografia e Estatística - IBGE. Síntese de Indicadores Sociais: uma análise das condições de vida da população brasileira. Rio de Janeiro: IBGE; 2010. (Informação Demográfica e Socioeconômica. n. 27).

6. Brasil. Lei no 8.842 , de 4 de janeiro de 1994. Dispõe sobre a política nacional do idoso, cria o Conselho Nacional do Idoso e dá outras providências. Diário Oficial da União, Brasília, p. 77, 1994 Jan 5, Seção 1.

7. Martins GA. Estatística geral e aplicada. $3^{\mathrm{a}}$ ed. São Paulo: Atlas; 2005.

8. Ministério da Saúde (BR), Coordenação Geral da Política de Alimentação e Nutrição. Orientações básicas para a coleta, processamento, análise de dados e informação em serviços de saúde para o sistema de vigilância alimentar e nutricional: versão preliminar. Brasília: Ministério da Saúde; 2004.

9. World Health Organization - WHO. Obesity: preventing and managing the global epidemic. Geneva: WHO, 1997. (Report of a WHO consultation on obesity).

10. Brasil. Resolução CNS no 466, de 12 de Dezembro de 2012. Aprova diretrizes e normas regulamentadoras de pesquisa envolvendo seres humanos. Diário Oficial da União, Brasília, 2013 Jun 13, n. 12, Seção 1, p. 59.

11. Kamijo CF. Prevalência de hipertensão e diabetes e taxa de mortalidade dos idosos do município de Marcelino Ramos (RS) [Dissertação de Mestrado]. Porto Alegre (RS): Pontifícia Universidade Católica do Rio Grande do Sul; 2010.

12. Victor JF, Ximenes LB, Almeida PC, Vasconcelos FF. Perfil sociodemográfico e clínico de idos atendidos em Unidade Básica de Saúde da Família. Acta Paul Enferm. 2009; 22(1):49-54.

13. Ministério da Saúde(BR), Departamento de Informática do SUS - DATASUS. Indicadores de fatores de risco e de proteção [Internet]. Brasília: Ministério da Saúde, 2010 [acesso em 2012 Abr 19]. Disponível em http:// tabnet.datasus.gov.br/cgi/tabnet.exe?idb2010/g02.def

14. Tavares DMS, Paiva MM, Dias FA, Diniz MA, Martins NPF. Socio-demographic characteristics and quality of life of elderly patients with systemic arterial hypertension who live in rural areas: the importance of nurses' role. Rev Latinoam Enferm. 2013; 21(2): 515-22.

15. Oliveira CJ, Moreira TMM. Caracterização do tratamento não-farmacológico de idosos portadores de hipertensão arterial. Rev Rene. 2010;11(1):76-85.

16. Giatti L, Barreto SM. Saúde, trabalho e envelhecimento no Brasil. Cad Saúde Pública. 2003;19(3):759-71.

17. Piati J, Felicetti CR, Lopes AC.Perfil nutricional de hipertensos acompanhados pelo Hiperdia em Unidade Básica de Saúde de cidade paranaense. Rev Bras Hipertens. 2009; 16(2):123-9.

18. Siqueira FV, Facchini LA, Piccini RX, Tomasi E, Thumé E, Silveira DS, et al. Atividade física em adultos e idosos residentes em áreas de abrangência de Unidades Básicas de Saúde de municípios das regiões Sul e Nordeste do Brasil. Cad Saúde Pública. 2008; 24(1):39-54.

19. Ferreira CCC, Peixoto MRG, Barbosa MA, Silveira EA. Prevalência de fatores de risco cardiovascular em idosos usuários do Sistema Único de Saúde de Goiânia. Arq Bras Cardiol. 2010; 95(5):621-8. 
20. Borba TB, Muniz RM. Sobrepeso em idosos hipertensos e diabéticos cadastrados no Sistema Hiperdia da Unidade Básica de Saúde do Simões Lopes, Pelotas, RS, Brasil. Rev Enferm Saúde. 2011;1(1):69-76.

21. Girotto E, Andrade SM, Cabrera MAS. Prevalência de obesidade abdominal em hipertensos cadastrados em uma Unidade de Saúde da Família. Arq Bras Cardiol. 2010; 94(6):754-62.

22. American Geriatrics Society. Mulheres correm mais riscos de serem obesas durante velhice. 2009 [acesso em 2012 Abr 3]. Disponível em http://www.bancodesaude. com.br/obesidade/107005052009-mulheres-corremmais-riscos-serem-obesas-durante-velhice

23. Krinski K, Elsangedy HM, Junior NN, Soares IA. Efeito do exercício aeróbio e resistido no perfil antropométrico e respostas cardiovasculares de idosos portadores de hipertensão. Acta Sci. Health Sci. 2006;28(1):71-5.

24. Brasil. Portaria n 2.982 , de 26 de novembro de 2009. Aprova as normas de execução e de financiamento da Assistência Farmacêutica na Atenção Básica. Diário Oficial da União, Brasília, p. 771-3, 2009 Nov 30, Seção1.

25. Contiero AP, Pozati MPS, Challouts RI, Carreira L, Marcon SS. Idoso com hipertensão arterial: dificuldades de acompanhamento na Estratégia Saúde da Família. Rev Gaúch Enferm. 2009; 30(1):62-70.
26. Araújo JC, Guimarães AC. Controle da hipertensão arterial em uma Unidade de Saúde da Família. Rev Saúde Pública. 2007;41(3):368-74.

27. Aguado A, López F, Miravet S, Oriol P, Fuentes MI, Henares B, et al. Hypertension in the very old; prevalence, awareness, treatment and control: a cross-sectional population-based study in a Spanish municipality. BMC Geriatrics. 2009;9:16.

28. Franklin SS, Khan SA, Wong ND, Larson MG, Levy D. Is pulse pressure useful in predicting risk for coronary heart disease? The Framingham Heart Study. Circulation. 1999;100(4):354-60.

29. Gusmão JL, Ginani GF, Silva GV, Ortega KC, Mion Junior D. Adesão ao tratamento em hipertensão arterial sistólica isolada. Rev Bras Hipertens. 2009;16(1): $38-43$.

\section{Endereço para correspondência}

Aluísio Oliveira de Andrade

Universidade Federal do Maranhão

Avenida dos Portugueses, 1966

Bairro: Bacanga

CEP: 65080-805 - São Luís - MA - Brasil

E-mail: aluisio12@hotmail.com 